\title{
ABORDAGEM ODONTOLÓGICA DE PACIENTE COM MICROSSOMIA HEMIFACIAL - RELATO DE CASO
}

\author{
Dental approach of a patient with hemifacial microssomia - case report
}

\author{
Tainara Steffens ${ }^{1}$, Letícia De Nardin²; Antônio Augusto Iponema Costa ${ }^{3}$
}

${ }^{1}$ Cirurgiã-dentista formada pela Universidade Regional Integrada do Alto Uruguai e das Missões - URI Erechim - RS.E-mail: tainara-steffens@hotmail.com

${ }^{2}$ Acadêmica do Curso de Odontologia da Universidade Regional Integrada do Alto Uruguai e das Missões - URI Erechim - RS.

${ }^{3}$ Docente do Curso de Odontologia da Universidade Regional Integrada do Alto Uruguai e das Missões - URI Erechim - RS.

Data do recebimento: 31/10/2019 - Data do aceite: 19/02/2020

RESUMO: A microssomia hemifacial é caracterizada como uma síndrome congênita em que ocorrem alterações no desenvolvimento embrionário do primeiro e segundo arcos branquiais. Sua etiologia ainda é desconhecida, todavia há evidências de origem genética. Há hipóteses de que possa advir de um acidente vascular no feto e que certos indivíduos apresentam suscetibilidades para desenvolver algumas malformações. O objetivo do trabalho foi relatar o caso clínico de um paciente com microssomia hemifacial. Na anamnese e exame clínico observou-se que o paciente possuía perda auditiva de 70\% do lado esquerdo e ausência dos elementos 51 e 61 decorrente de traumatismo dentário, deglutição normal, respiração predominantemente nasal, possuía interposição de língua, fonação atípica, contração do mentalis, presença de ação labial e mordida aberta. O paciente tinha como características, sendo tipo I de Baume superior, espaços primatas na arcada superior e inferior, bem como análise facial padrão II. O tratamento proposto foi instalação de um aparelho ortodôntico estético e funcional. Uma avaliação cuidadosa da morfologia craniofacial pelo ortodontista é de extrema importância para referenciar precocemente os casos em que seja necessária uma intervenção terapêutica, por meio de uma abordagem multiprofissional que busque melhorar a aparência e funcionalidade das estruturas acometidas.

Palavras-chave: Assimetria Facial. Ortodontia. Qualidade de Vida. 


\begin{abstract}
Hemifacial microsomia, a congenital syndrome, is characterized by changes in the embryonic development of the first and the second branchial arches. Its etiology is still unknown, but there is strong evidences of genetic origin. There are chances that it may result from a stroke in the fetus and that certain individuals are susceptible to developing some malformations. The aim of this study was to report a clinical case of hemifacial microsomia. It was observed, in the anamnesis and clinical examination that the patient had $70 \%$ hearing loss on the left side and absence of elements 51 and 61 due to dental trauma, normal swallowing, predominantly nasal breathing, tongue interposition, atypical phonation, mentalis contraction, presence of lip action and open bite. The patient's predominant characteristics were Baume type I and primate spaces in the upper and lower arches, as well as standard facial analysis type II. The proposed treatment was the use of an aesthetic and functional orthodontic appliance. A careful evaluation of craniofacial morphology by the orthodontist is extremely important to refer early to cases in which therapeutic intervention is necessary, through a multiprofessional approach that seeks to improve the appearance and functionality of the affected structures.
\end{abstract}

Keywords: Facial Asymmetry. Orthodontics. Quality of Life.

\section{Introdução}

De acordo com Gorlin et al. (1963), a microssomia hemifacial é caracterizada como uma síndrome congênita em que ocorrem alterações no desenvolvimento embrionário do primeiro e segundo arcos branquiais e o grau de comprometimento apresenta-se desde um pequeno acometimento do osso temporal ou da cabeça da mandíbula até uma fissura orbital nos casos mais graves (RIBEIRO, 2011).

Diferentes nomenclaturas têm sido utilizadas para denominar essa entidade, sendo elas: Microssomia Hemifacial, Microssomia Craniofacial, Displasia Óculo-Aurículo-Vertebral, Síndrome de Goldenhar-Gorlin, Síndrome do Primeiro e Segundo Arcos Branquiais e Displasia Facial Lateral (PINTO, 2004). O termo Displasia Óculo-Aurículo-Vertebral foi sugerido em casos que incluem anomalias vertebrais, objetivando fornecer uma caracterização mais abran- gente a essa síndrome tão variada em sua apresentação e etiopatogenia (GORLIN et al., 1963).

A causa da síndrome ainda não é bem conhecida; há evidências de origem genética, com transmissão autossômica dominante, na qual a morfologia da crista neural seria afetada, ocasionando malformação dos derivados do primeiro e segundo arcos branquiais, afetando face, orelhas, olhos, vértebras e causando outras anomalias (REGENBOGEN et al., 1982). Há hipótese de que a síndrome possa advir de um acidente vascular no feto, causando a diminuição de aporte sanguíneo e produção de coágulos na área daqueles tecidos que desenvolverão as estruturas da orelha e mandíbula. A severidade do dano causado pelo acidente vascular estaria diretamente relacionada ao período de desenvolvimento em que este aconteceu, bem como à área de destruição que houve no tecido afetado (LAM, 2000). Outra teoria é que certos indivíduos apresentam 
suscetibilidade para desenvolver algumas malformações, baseadas na ocorrência de mutações em genes receptores de folato, que podem aumentar o risco de aparecimento de deformidades congênitas mandibulofaciais (QUIRÓS; SATURNO, 2003).

A microssomia hemifacial é a segunda anomalia congênita facial que mais acomete a população (1 em cada 5.600 nascidos vivos), logo após a fissura labial e palatina. Esta alteração geralmente é observada unilateralmente e causa uma deformidade facial caracterizada por diminuição do corpo da mandíbula e desvio do mento para o lado afetado. Do lado contralateral, temse um alongamento da mandíbula e a face é achatada (BUENO, 2002). As pessoas com essa síndrome apresentam anomalias bucais, musculoesqueléticas, auriculares e oculares, alterações cardíacas, genitais, renais, pulmonares e de sistema nervoso central (SILVA et al., 2003).

Poswillo (1974) relata que o tratamento das crianças com a Síndrome de Goldenhar deveria obedecer a um planejamento cronológico e ser efetuado por equipe multidisciplinar, que daria prioridade aos eventos de maior gravidade e que poderiam influir nos aspectos respiratórios, auditivos e funcionais do paciente.

Diante do exposto, este trabalho teve como objetivo relatar o caso clínico referente às repercussões odontológicas oriundas da Microssomia Hemifacial, na perspectiva do diagnóstico ao tratamento com vista à melhoria da qualidade de vida.

\section{Relato do Caso Clínico}

O presente relato de caso clínico possui um acompanhamento de 2 anos e 6 meses. Obteve aprovação do Comitê de Ética em Pesquisa da Universidade Regional
Integrada do Alto Uruguai e das Missões, sob o parecer número 1.888.542.

Paciente E.C.A, sexo masculino, com diagnóstico de Microssomia Hemifacial, foi encaminhado para a Clínica-Escola de Odontologia no Centro de Estágios e Práticas Profissionais da Universidade Regional Integrada do Alto Uruguai e das Missões - URI Erechim (URICEPP), para atendimento odontológico na disciplina de odontopediatria. Quando encaminhado para seu primeiro atendimento, possuía 3 anos e 9 meses de idade.

No primeiro contato com o paciente foi realizada anamnese e exame clínico, em que constatou-se que o paciente apresentava perda auditiva severa do lado esquerdo, ausência dos elementos 51 e 61 decorrente de um traumatismo dentário, e também apresentava hábitos deletérios, como chupar o dedo e chupeta. Além disso, foi diagnosticado com deglutição normal, respiração predominantemente nasal, interposição de língua, fonação atípica, contração do músculo mentalis presente, não possuía selamento labial e sua mordida era aberta. As Figuras 1, 2 e 3 mostram as fotografias do paciente, ficando evidentes as alterações faciais.

O paciente já consultava com fonoaudióloga em escolas estaduais do município por um determinado tempo. Conforme relatos da mãe, realizou consultas de pré-natal, sendo que o paciente nasceu de parto cesariana pesando $3.335 \mathrm{~kg}$. Ao nascer, foram realizados exames como tomografia computadorizada para avaliar a alteração facial e regiões adjacentes, sendo necessário acompanhamento por tempo indeterminado. Em razão da falta de vedamento labial, não foi possível realizar o aleitamento materno e, por isso, fez uso de mamadeira.

Antes do paciente iniciar os acompanhamentos odontológicos, foi encaminhado 
Figura 1 - Visão frontal

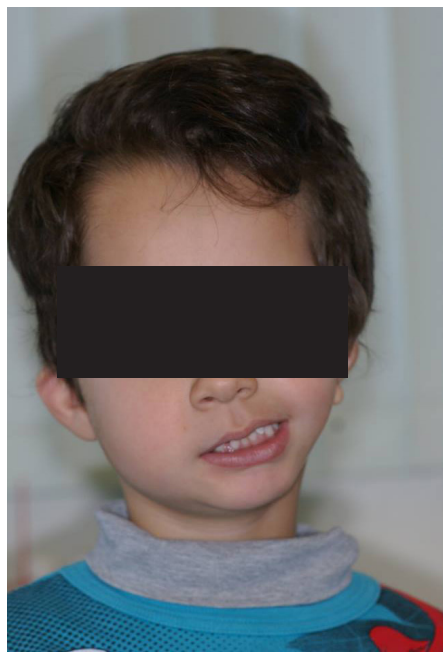

Figura 2 - Lado direito

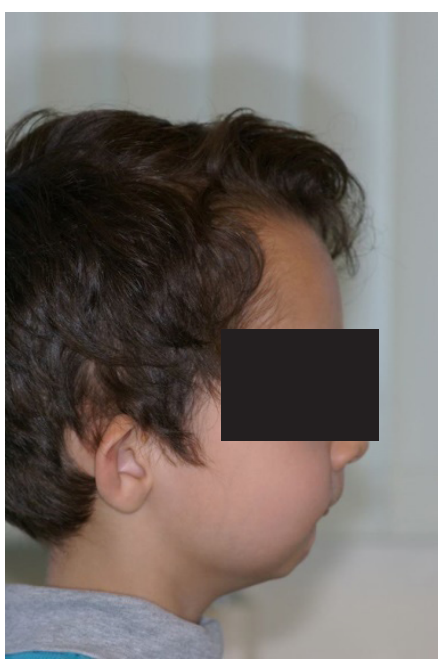

Figura 3 - Lado esquerdo

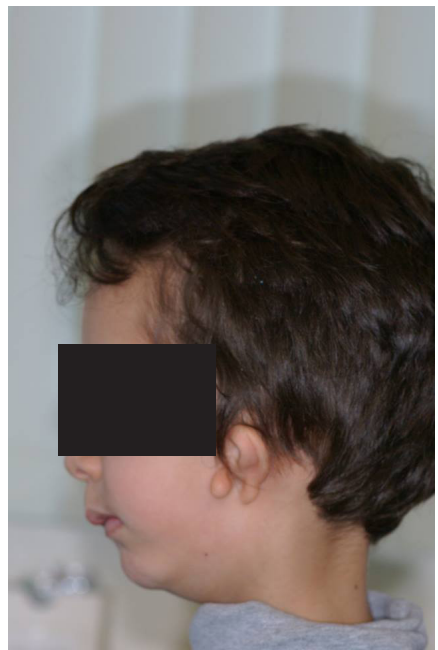

para avaliação no Hospital da Criança e, em seguida, para o Hospital de Clínicas, no município de Porto Alegre (RS), onde foram solicitados exames radiográficos e tomografia computadorizada, porém os exames não foram disponibilizados aos autores do artigo.

Na primeira sessão de atendimento odontológico, conversou-se com a mãe e foi apresentado o ambiente ao paciente, que se mostrava bastante ansioso. Realizou-se a anamnese, exame clínico, fotografias intra e extrabucais, moldagem com alginato nas arcadas superior e inferior e solicitação de exames radiográficos.

Após atendimento na disciplina de odontopediatria, quando foi feita a adequação do meio bucal do paciente, o mesmo foi encaminhado para avaliação na disciplina de ortodontia preventiva. Nesta optou-se, como forma de tratamento, pelo aparelho ortodôntico estético e funcional, devido à ausência dos elementos dentários 51 e 61 . Observou-se que a dentição apresentava atraso na sua erupção, o que pode ser uma característica clínica da síndrome. O acompanhamento odontológico nessa situação é de extrema importância, dando atenção à preservação do espaço para a erupção da dentição permanente (Figuras 4 e 5).

No exame intrabucal, o paciente foi diagnosticado com o arco dentário tipo I de Baume superior, ou seja, presença de espaços localizados entre os molares na dentição decídua. Também possuía espaços primatas na arcada superior e inferior, ou seja, espaço que se localiza entre os caninos e primeiros molares decíduos na arcada inferior e entre os incisivos laterais e caninos na arcada superior. Com relação à análise facial, foi considerado como padrão II.

No decorrer dos anos de 2015 e 2016 , o paciente continuou em atendimento e acompanhamento odontológico. O mesmo foi encaminhado para realização de exame radiográfico do tipo panorâmico (Figura $6)$, telerradiografia frontal (PA) e lateral, sendo diagnosticada a agenesia no côndilo mandibular, desvio de septo nasal e assimetria mandibular acentuada. Além disso, foi encaminhado para realizar tomografia computadorizada por meio do Sistema Único de Saúde (SUS), no município de Erechim, sendo encaminhada pela Secretaria Municipal de Saúde (SMS). 
Figura 4 - Mordida aberta e cruzada

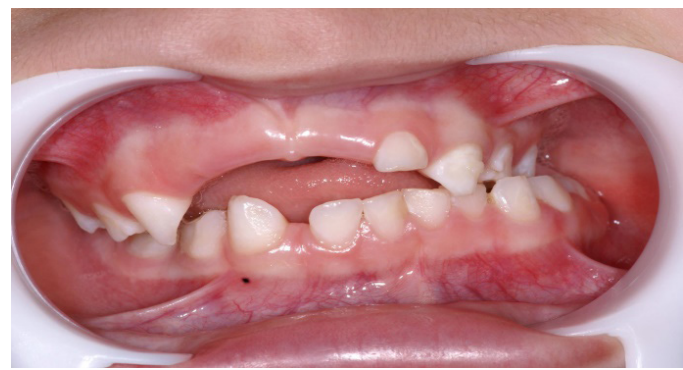

Figura 5 - Utilização do aparelho ortodôntico

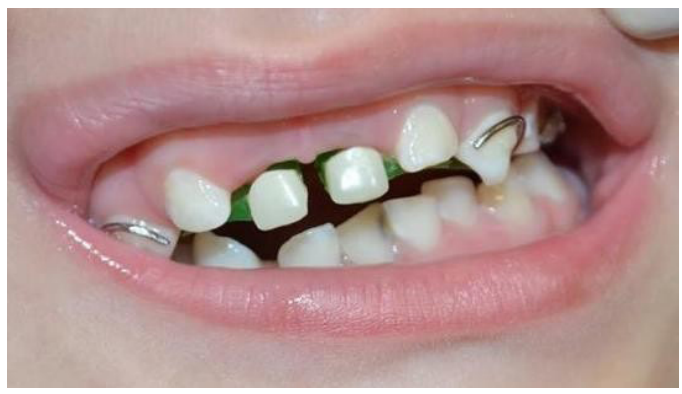

Durante o atendimento, foi aplicado um formulário adaptado do Child Perceptions Questionnaire 8-10 (CPQ 8-10) ao responsável, com perguntas relacionados ao convívio do paciente com outras crianças e de como ele reagia a reações diferentes. As perguntas eram objetivas, e não houve dificuldade para respondê-las.
Ao passar pela disciplina de ortodontia preventiva no ano de 2016, em planejamento feito com professores, chegou-se ao consenso de que não haveria mais necessidade do paciente realizar tratamento ortodôntico com aparelhos removíveis, pois a sua desoclusão impedia a adaptação do aparelho, e foi sugerido um acompanhamento periódico do paciente, que foi orientado a retornar a cada 3 meses, a fim de observar o atraso na erupção.

O caso foi avaliado e discutido por uma equipe composta por professores do curso de Odontologia, e optou-se por encaminhar o paciente novamente para o Hospital de Clínicas de PortoAlegre, RS, a fim de continuar o tratamento de expansão da mandíbula. Este tratamento cirúrgico diminuiria a assimetria facial e desoclusão entre as arcadas superior e inferior.

\section{Discussão}

O paciente em estudo continua em acompanhamento na Clínica-Escola de Odontologia da URI Erechim.

Da mesma forma como a literatura tem referenciado, agenesia condilar causa uma deformidade facial caracterizada por dimi-

Figura 6 - Radiografia panorâmica evidenciando a agenesia do côndilo mandibular (esquerdo)

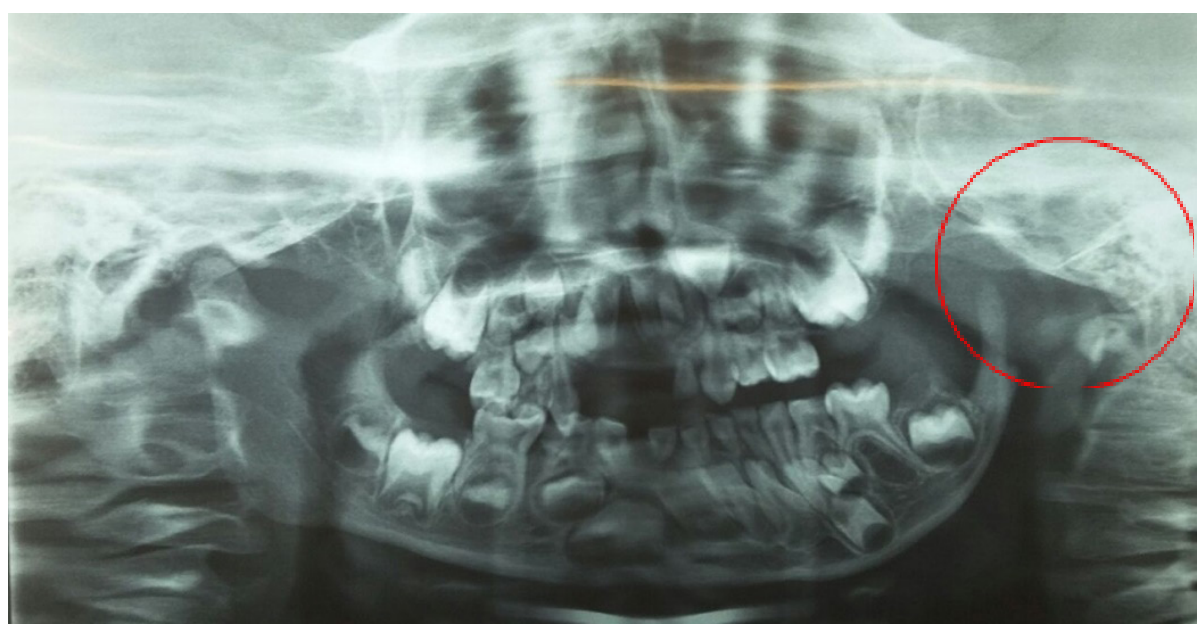


nuição do corpo da mandíbula e desvio do mento para o lado afetado, enquanto no lado contralateral observa-se um alongamento da mandíbula e a face é achatada (BUENO, 2002). Neste caso, o paciente possuía um desvio de mento para esquerda e alongamento da mandíbula do lado direito. Ishytoia et al. (1997) apud Ribeiro (2011) afirmam que as pessoas com essa síndrome podem apresentar graus variados de alguns ou todos os sinais relatados.

As peculiaridades desta síndrome foram evidentes neste paciente, visto que apresentava comprometimento do pavimento auricular do lado esquerdo, bem como perda auditiva de $70 \%$. A literatura tem corroborado, relatando que pacientes com microssomia hemifacial, além das malformações, possuem características que irão influenciar em suas funções respiratória, auditiva e funcional (QUIRÓS; SATURNO, 2003). Além disso, a face apresenta, no conjunto, características visuais específicas, devido à assimetria facial, hipoplasia e deslocamento do pavilhão auricular, bem como do comprometimento do osso malar e temporal, com envolvimento dos maxilares, resultando no seu achatamento devido à aplasia ou hipoplasia do ramo da mandíbula e do côndilo (PINTO, 2004).

A língua pode ser hipoplásica na musculatura do lado afetado com malformação complexa, unilateral, onde a assimetria facial está presente; os ossos maxilar, temporal e zigomático podem se apresentar reduzidos no tamanho e em espessura (GREENWOOD, et al., 1982).

Com relação à oclusão, é comum encontrar mordida cruzada do lado afetado devido ao desvio da mandíbula para este lado. Criase, então, uma maxila assimétrica com o processo alveolar pouco desenvolvido do lado cruzado e, consequentemente, estará presente um desvio de linha média considerável para o lado afetado e, possivelmente, uma sobremordida profunda devido a ações musculares (KAPLAN, 1989). Essa desoclusão foi diagnosticada no paciente, que apresentou dificuldades na adaptação e estabilização de um aparelho removível estético e funcional, com a finalidade principal de restabelecer a função dos elementos perdidos e suprir as necessidades estéticas. Em relação aos aspectos estéticos do paciente, houve grande solicitação por parte da família, que lhe incentivou o uso do dispositivo, porém o paciente não conseguiu adaptar-se pelo desconforto que o mesmo causava.

Alterações cardiovasculares têm sido descritas em 5 a $58 \%$ dos pacientes com essa síndrome, sendo que o prognóstico dessa condição depende, frequentemente, das condições cardiovasculares subclínicas (BRIEGAR et al., 1998). Pacientes que possuem microssomia hemifacial podem apresentar, também, anormalidades renais e ureterais, podendo acarretar obstrução do trato urinário, com posterior dilatação pielocalicial e insuficiência renal (RIBEIRO et al., 2011). Entretanto, estas alterações não foram diagnosticadas no paciente em estudo.

Em relação ao sexo, a literatura aponta pequena variação, sendo mais comum em mulheres. Pacientes que apresentam síndromes com características de malformações craniofaciais, principalmente aquelas com manifestações na cavidade bucal, além do diagnóstico precoce, devem ser tratados obedecendo a um rigoroso planejamento cronológico e por equipe de saúde interdisciplinar extremamente integrada, que deverá dar prioridade aos eventos de maior gravidade (KAHL-NIEKE; FISCHBACH, 1998).

A técnica recomendada para casos de assimetrias severas é a cirurgia precoce. A partir dos três anos de idade está indicada a retirada de tecido esterno clavicular para ser enxertado, posteriormente, e fixado com miniplacas de titânio na mandíbula, objetivando que facilite o desenvolvimento 
ósseo facial, restabelecer a simetria facial e reparar as deformidades faciais (QUIRÓS; SATURNO, 2003). O paciente relatado é monitorado desde seu nascimento, com acompanhamento multidisciplinar de cirurgião-dentista (clínico geral), cirurgião bucomaxilofacial, médicos, fonoaudióloga e psicóloga. Conforme a família, segundo orientações médicas, aguarda-se o paciente ter 6 anos de idade para realizar a cirurgia de alongamento da mandíbula.

A literatura institui o tratamento cirúrgico com aumento do lado hipoplásico seguido de tratamento ortodôntico, para estabelecer uma oclusão dentária normal (BUENO, 2002). Para Yamashi et al. (1997), para pacientes com deformidades severas indica-se intervenção cirúrgica precoce com transplante autógeno costocondral ou, posteriormente, cirurgia ortognática combinada de maxila e mandíbula. Entretanto, a prioridade, neste relato de caso, foi descrever as intervenções odontológicas em nível ambulatorial, sempre levando em consideração suas complexidades e primando pelo bem-estar do paciente. Neste sentido, Kahl-Nieke e Fischbach (1998) destacam a importância da melhora na qualidade de vida dos pacientes a partir de intervenções multiprofissionais.

Para Simões (2003), a terapia ortopédica em fase precoce mostra-se efetiva, já que na microssomia hemifacial o problema não é somente esqueletal, mas também muscular, e outros elementos de tecido mole possivelmente estão alterados. Com a estimulação do aparelho funcional é possível obter algum desenvolvimento de tecidos moles e, desta maneira, melhorar o desenvolvimento facial e o prognóstico em caso de necessidade de uma correção cirúrgica. Além disso, na maioria dos casos necessita-se estimular o crescimento transversal da maxila para a correção da mordida cruzada posterior (RIBEIRO et al., 2011).

No caso relatado por Ribeiro et al. (2011) sobre um caso clínico de uma criança de qua- tro anos e nove meses de idade com ausência congênita do côndilo esquerdo, inicialmente foi utilizado um aparelho ortopédico funcional, também preconizado por Simões (2003). Após descruzar a mordida e reposicionar a mandíbula anteriormente, foi utilizado um segundo aparelho, com a função de corrigir o plano oclusal e manutenção da oclusão alcançada. Os autores observaram uma melhora no perfil facial devido ao avanço mandibular, melhora na assimetria facial e melhora na oclusão dentária, ocorrendo descruzamento dentário, correção de linha mediana inferior e correção da Classe II dentária do lado esquerdo.

A assimetria facial é consequência de algum crescimento desordenado das estruturas craniofaciais, podendo ser desencadeada por fatores genéticos, fatores ambientais como hábitos e/ou traumas, malformações congênitas e desvios funcionais, que acabam por comprometer o crescimento mandibular (LEGAN, 1998). Em graus mais severos, além de acometer a estética, a assimetria mandibular pode afetar a função (BISHARA; BURKEY; KHAROUF, 1994). A simetria e o equilíbrio, quando aplicados à morfologia facial, proporcionam harmonia e padrão entre as estruturas faciais (PROCACI; RAMALHO, 2002).

$\mathrm{O}$ diagnóstico dado à assimetria vem após um minucioso exame clínico e anamnese, exame físico, radiográfico, com dados específicos e detalhados, que possam auxiliar no decorrer do tratamento relacionado aos problemas bucais e também de outras áreas. Dahan (2001) destaca que os exames complementares são fundamentais no estabelecimento do diagnóstico definitivo, como a radiografia panorâmica, a radiografia posteroanterior (PA) de crânio e mandíbula, a tomografia computadorizada em cortes coronais e axiais e a radiografia de perfil, que auxilia no diagnóstico de desnivelamento da 
porção basilar da mandíbula. Já o exame físico, segundo Yoon e Kim (2002), é realizado fazendo-se comparações entre as duas hemifaces, verificando diferenças de tamanho e forma. Observa-se também se há presença de desvios da mandíbula, limitação na abertura bucal e desvios do mento. Benson e Laskin (2001) sugerem a utilização de espátulas de madeira, sendo que o paciente apreende entre os dentes a espátula e mensura-se a distância entre a espátula (na altura da comissura labial) até a linha interpupilar, diagnosticando clinicamente o envolvimento ou não da maxila na assimetria. Observou-se, no paciente em estudo, a partir de exames complementares de imagem (radiografia panorâmica), que o mesmo apresentava agenesia de côndilo da mandíbula do lado esquerdo.

Peterson et al. (2000) enfatizam que a avaliação deve incluir o osso frontal, os olhos, os rebordos infraorbitários, as eminências zigomáticas, asa do nariz, as áreas paranasais, morfologia do lábio, o relacionamento dos lábios com os incisivos e todo o relacionamento proporcional da face nos sentidos vertical e transversal.

\section{Considerações Finais}

Pode-se perceber que não há um consenso na literatura quanto à terminologia adequada para se referir a essa condição. Alguns artigos citam a Microssomia Hemifacial como sinônimo de Síndrome Goldenhar, outros tratam esse termo como uma das alterações componentes da síndrome.
A avaliação cuidadosa da morfologia craniofacial pelo cirurgião dentista é de extrema importância para referenciar precocemente os casos em que seja necessária uma intervenção terapêutica. Conclui-se que o tratamento para microssomia facial deve ser determinado individualmente, por meio de uma abordagem multiprofissional, buscando melhorar a funcionalidade das estruturas acometidas, bem como a estética, levando sempre em consideração as necessidades de cada paciente.

É necessário que o cirurgião-dentista tenha conhecimento das características clínicas e sistêmicas de qualquer malformação, sendo de suma importância proporcionar ao paciente tratamento precoce adequado, tendo conhecimento de todo caso, tornando, assim, mais viável manter relação interdisciplinar, orientação ao paciente, bem como intervenções relacionadas à área de atuação de cada profissional, com intuito de melhorar e manter a qualidade de vida do paciente com a síndrome. Foi notável a preocupação da família em relação aos cuidados com a saúde bucal e sistêmica do seu filho, mostrando-se interessados, permitindo que o tratamento fosse realizado com maior efetividade. Essa relação família-paciente-profissional foi de extrema importância para o sucesso durante o tratamento.

A Microssomia Hemifacial ainda é assunto escasso dentro da odontologia e das plataformas de busca científica. Mais estudos clínicos observando a etiopatogenia e o desenvolvimento da síndrome são necessários para entender melhor seu prognóstico em longo prazo. 


\section{REFERÊNCIAS}

BENSON, K. J.; LASKIN, D. M. Upper lip asymmetry in adults during smiling. J Oral Maxillofac Surg, v. 59, p. 396-398, 2001.

BISHARA, S.E.; BURKEY, P.S.; KHAROUF, J.G. Dental and facial asymmetries: a review. Angle Orthod., v. 64, n. 2, p. 89-98, 1994.

BRIEGAR, P. et al. Oculo-auriculo-vertebral disorder (Goldenhar syndrome) coexisting with schizophreniform disorder. J. Neurol. Neurosurg. Psychiatr., v. 65, n. 1, p. 135-136, 1998.

BUENO DF. Estudo das alterações clínicas e radiográficas da microssomia hemifacial [dissertação]. São Paulo: Universidade de São Paulo; 2002.

DAHAN, J. A simple digital procedure to assess facial asymmetry. Am J Orthod Dentofacial Orthop, v. 122, n. 1, p. 110-116, 2001.

GORLIN, R.J. et al. Oculoauriculovertebral dysplasia. J Pediatr., v. 63, p. 991-999, 1963.

GREENWOOD, F.M. et al. Further evidence for an autossomal dominant form of oculoauriculovertebral dysplasia. Clin. Genet., v. 21, n. 3, p. 161-167, 1982.

ISHITOYA, S. et al. Left retrocaval ureter associated with the Goldenhar Syndrome (Branchial Arch Syndrome). J. Urol., v. 158, n. 2, p. 572-573, 1997.7

KAHL-NIEKE B, FISCHBACH R. Effect of early orthopedic intervention on hemifacial microsomia patients: an approach to a cooperative evaluation of treatment results. Am J Orthod Dentofac Orthop. v. 114, n.5, p.538-550, 1998.

KAPLAN, R.G. Induced condylar growth in a patient with hemifacial microsomia. Angle Orthod., v. 59, n. 2, p. 85-90, 1989.

LEGAN HL. Surgical correction of patients with asymmetries. Semin Orthod., v. 4, n. 3, p. 189-98, 1998.

LAM, C.H. A theory on the embryogenesis of oculo-auriculo-vertebral (Goldenhar) syndrome. J

Craniofacial Surg., v. 11, n. 6, p. 547-552, 2000.

PETERSON, L. J. et al. Cirurgia oral e maxilofacial contemporânea. 3. ed. Rio de Janeiro: Guanabara Koogan, 2000.

PINTO, N.F. et al. Relato de caso clínico - Síndrome de Goldenhar. Publ. UEPG Ci. Biol. Saúde, v. 10, n. 3/4, p. 25-32, 2004.

POSWILLO, D. Otomandibular deformity: pathogenesis as a guide to reconstruction. J Maxillofac Surg, v. 2, n. 3, p. 64-72, 1974.

POSWILLO, D. The pathogenesis of the first and second branchial arch syndrome. Oral Surg Oral Med Oral Pathol., v. 53, n. 3, p. 302-328, 1973.

PROCACI, M.I.M.A.; RAMALHO, S.A. Crescimento assimétrico da face: atividade muscular e implicações oclusais. Rev. Dental Press Ortod Orthop Facial, v. 7, n. 6, p. 87-93, 2002.

QUIRÓS, O.; SATURNO, L.E. Agenesia del cóndilo, crecimiento de cóndilo suplementario em paciente tratado com ortopedia funcional de los maxilares, sin cirugía. Rev. Latinoam Ortod Odontop., 2003.

REGENBOGEN, L. et al. Further evidence for an au-tosomal dominant form of oculoauriculovertebral dysplasia. Clin Genet, v. 21, n. 3, p. 161-167, 1982. 
RIBEIRO, F.A.V. et al. Tratamento não cirúrgico de microssomia hemifacial por meio da ortopedia funcional dos maxilares. RGO, v. 59, n. 1, p. 131-134, 2011.

SIMÕES, W.A. Ortopedia funcional dos maxilares vista através da reabilitação neuro-oclusal. 3. ed. São Paulo: Artes Médicas; 2003.

SILVA, L.C.P.; PENIDO, C.V.S.R. Síndrome de Goldenhar: relato de caso clínico. J Bras

Odontopediatr Odontol Bebe, v. 6, n. 32, p. 283-286, 2003.

YAMASHIRO, T. et al. Case report: dentofacial orthopedic and surgical orthodontic treatment in hemifacial microsomia. Angle Orthod., v. 67, n. 6, p. 463-466, 1997.

YOON, H. J.; KIM, H. G. Intraoral mandibular distraction osteogenesis in facial asymmetry patients with unilateral temporomandibular joint bony ankylosis. Int J Oral Maxillofac Surg, v. 31, p. 544$548,2002$. 